\title{
Sustained mismatching performance in pigeons with chronically maintained conditioned reinforcement
}

\author{
JOSEPH ZIMMERMAN and PETER V. HANFORD \\ Indiana University School of Medicine and Indiana University \\ Purdue University at Indianapolis, Indianapolis, Indiana 46202
}

\begin{abstract}
Pigeons were presented with free grain via a variable-interval tape if or after no pecking had occurred for 2 sec. Concurrently, mismatching-to-sample performance intermittently produced conditioned reinforcement in only one component of a two-component multiple schedule. Throughout a series of experimental manipulations, mismatching was differentially sustained in the conditioned-reinforcement component. Performances were relatively insensitive to changes in the frequency of free gain delivery over a wide range of frequencies. These results extend previous results to complex discrimination and suggest that the frequency of sustained responding is relatively independent of the frequency of free grain.
\end{abstract}

In a series of studies in this laboratory, Zimmerman and Hanford (1966) and Zimmerman (1969) demonstrated that keypecking in pigeons could be maintained indefinitely at moderate rates by the response-contingent presentation of stimuli paired with the delivery of free grain. In these studies, the strength of the conditioned reinforcing stimuli (CR) was maintained by "free grain" which was delivered concurrently on a variable-interval reinforcement schedule. "Accidental" reinforcement of keypecking was prevented by delaying the delivery of free grain, when primed, until or unless there had been no keypeck in the preceding $6 \mathrm{sec}$. The use of control procedures including the removal of $C R$, the replacement of $C R$ with a set of neutral stimuli, and the removal of free grain, demonstrated that the maintained behavior could only be attributed to conditioned reinforcement.

In the above studies, the response that was maintained was pecking a single key. The present study was an extension of the preceding studies. Its purpose was to determine whether $\mathrm{CR}$ could similarly maintain performance of a more complex three-key task, mismatching to sample. This procedure differs from matching-to-sample (Ferster, 1960; Zimmerman \& Ferster, 1963) in that in mismatching to sample, a center key is illuminated with one of two alternate colored lights. A peck on the center key turns off the center keylight (sample) and illuminates the two side keylights. One of these side keys has the original sample color projected upon it while the other has the alternate color projected on it. A correct mismatching response is defined as pecking the side key whose color does not match the center-key sample stimulus. In the present experiment, mismatching to sample was reinforced on an intermittent basis by CR.

\section{EXPERIMENT I}

\section{Method}

Subjects. Three adult male White Carneaux pigeons, each with extensive previous experience (from 2 months to 5 years) in matching-to-sample and mismatching-to-sample experiments participated in this study. Their body weights were reduced to $80 \%$ of their free-feeding weights, and each subject performed daily in a standard three-key experimental space.

Apparatus and procedure. Standard electromechanical equipment housed in an adjacent room was used to program the contingencies and record the performances of the subjects.

Throughout each daily $90-\mathrm{min}$ session, $2 \frac{1}{2}-\mathrm{sec}$ grain presentations were freely delivered via a 2 -min variable-interval (VI) tape, provided that no pecking on any of the three keys and no stimulus event (CR or blackout) had occurred for at least $2 \mathrm{sec}$. The magazine cycle was accompanied by the illumination of the magazine light, and the sound of the solenoid-operated magazine. During this cycle, the houselights and center keylight remained illuminated. The $\mathrm{CR}$ used in this study consisted of $2 \frac{1}{2} \mathrm{sec}$ of the above stimulus changes. That is, the magazine light was illuminated, the center keylight for the next sequence was turned on, and the magazine solenoid was energized. The houselights remained on throughout the CR cycle. During the $2 \frac{1}{2}$-sec CR cycle, the subjects were prevented from obtaining grain by a shutter which remained over the opening to the grain (Leckrone, Zimmerman, \& Hanford, 1966).

The pigeons were exposed to a two-component multiple schedule of mismatching to sample. The two components were differentially signaled by the presence of white noise in the first component and a $400-\mathrm{Hz}$ tone in the second. In both components, incorrect (matching) sequences resulted in a $2^{1 / 2}-\mathrm{sec}$ blackout. During the blackout, the houselights and keylights were turned off. Each component of the multiple schedule was $5 \mathrm{~min}$ in duration, and the components alternated over the duration of the session. Under the first (baseline) condition, correct (mismatching) sequences intermittently produced CR on a VI 1-min schedule in both components. When behavior stabilized (four sessions with no consistent trend in mismatching rates), CR was removed from thefirst component. Over the course of two additional manipulations, the reinforcement contingencies associated with the two components were reversed.

\section{Results}

Table 1 presents: (a) the median mismatching sequence rates in each component, (b) the number of sessions of exposure to each condition for each subject, and (c) the median mismatching accuracies (the ratio of mismatching sequences divided by the total sequences) 
Table 1

Experimental Conditions and Results

\begin{tabular}{|c|c|c|c|c|c|c|c|c|c|c|c|c|c|c|c|}
\hline \multirow[b]{3}{*}{ FOOD } & \multicolumn{4}{|c|}{$27 \mathrm{~B}$} & \multicolumn{4}{|c|}{$83 \mathrm{Y}$} & \multicolumn{4}{|c|}{$7 \mathrm{~B}$} & \multirow{2}{*}{\multicolumn{3}{|c|}{$\begin{array}{c}\text { Number of } \\
\text { Sessions }\end{array}$}} \\
\hline & \multicolumn{2}{|c|}{ CR COMP } & \multicolumn{2}{|c|}{$\begin{array}{c}\text { NON } \\
\text { CR COMP }\end{array}$} & \multicolumn{2}{|c|}{ CR COMP } & \multicolumn{2}{|c|}{$\begin{array}{c}\text { NON } \\
\text { CR COMP }\end{array}$} & \multicolumn{2}{|c|}{ CR COMP } & \multicolumn{2}{|c|}{$\begin{array}{c}\text { NON } \\
\text { CR COMP }\end{array}$} & & & \\
\hline & MM & Acc & MM & Acc & $\mathrm{MM}$ & Acc & MM & Acc & MM & Acc & MM & Acc & $27 \mathrm{~B}$ & $83 Y$ & $7 \mathrm{~B}$ \\
\hline VI 2 & 9.5 & .92 & $8.3 *$ & .91 & 3.1 & .88 & $3.4^{*}$ & .90 & 7.3 & .90 & $6.9^{*}$ & .89 & 16 & 17 & 17 \\
\hline VI 2 & 8.8 & .84 & 4.1 & .74 & 2.7 & .81 & 1.7 & .76 & 4.5 & .65 & 1.8 & .58 & 25 & 24 & 25 \\
\hline VI 2 & 9.2 & .78 & 3.3 & .81 & 2.1 & .79 & 1.2 & .79 & 6.6 & .87 & 2.3 & .84 & 24 & 24 & 24 \\
\hline VI 2 & 10.5 & .75 & 4.1 & .73 & 4.2 & .74 & 1.0 & .77 & 8.3 & .86 & 2.2 & .80 & 7 & 10 & 12 \\
\hline VI 6 & 11.7 & .79 & 3.2 & .73 & 5.1 & .73 & 1.5 & .64 & 9.8 & .87 & 3.1 & .78 & 8 & 8 & 6 \\
\hline VI 12 & 11.8 & .70 & 2.4 & .71 & 5.0 & .70 & .96 & .70 & 12.3 & .87 & 4.2 & .79 & 10 & 11 & 6 \\
\hline VI 18 & 15.2 & .75 & 3.4 & .75 & 4.4 & .69 & .94 & .68 & 11.8 & .88 & 2.0 & .77 & 11 & 7 & 12 \\
\hline EXT & 9.1 & .72 & 2.0 & .74 & .98 & .61 & .23 & .63 & 2.4 & .89 & .96 & .82 & 9 & 7 & 7 \\
\hline
\end{tabular}

*In the initial condition $C R$ was presented in both components for all subjects.

Code: FOOD-21/2-sec cycles of free food presented on a VI schedule as indicated; EXT-Free food cycles are absent; CR COMP-Correct sequences produce 21/2-sec CR cycles on a 1-min VI schedule; NON CR COMP-CR cycles are not presented in this component; MM-Median mismatching sequence rate (based on last five sessions); Acc-Median mismatching accuracy (mismatching sequences/total sequences).

in each component for each of the three birds.

Table 1 shows that mismatching was sustained at moderate rates with $\mathrm{CR}$ throughout this experiment. Mismatching rates in the CR components always markedly exceeded corresponding rates in the components from which $\mathrm{CR}$ was absent. Finally, mismatching accuracy always exceeded .50 , indicating that mismatching rates in each component always exceeded matching rates, regardless of whether these did or did not produce CR.

Although mismatching rates increased and declined, respectively, when $\mathrm{CR}$ was introduced or removed, these results differed from previous results obtained in this laboratory. Zimmerman and Hanford (1966), for example, found that the removal of the CR resulted in near extinction of responding. Mismatching may have been maintained in the non-CR components by stimulus and response generalization since conditions in both components were extremely similar with respect to the appearance of the center and side key colors on a response-contingent basis.

\section{EXPERIMENT II}

Subjects and apparatus were the same as used in Experiment I.

Zimmerman (1969), using a single-key concurrent procedure, manipulated the frequency of free grain presentation from $1 \mathrm{~min}$ to $18 \mathrm{~min}$ and extinction. $\mathrm{He}$ found that performance maintained by CR was reduced only at the extremes of free grain presentation (VI $1 \mathrm{~min}$ and EXT). Over the range of values between the extremes (2-min to 18-min, variable interval schedule) no systematic change in the performance maintained by CR was observed.

Experiment II of the present study similarly examined the effects of varying the frequency of free food on the strength and accuracy of mismatching performance. The same three subjects were exposed to a multiple schedule in which CR was programmed only in the second component for two subjects and in the first component for the third subject (Bird 27B). The frequency of free grain delivery was programmed on either a 1 -min, a 2-min, a 6-min, a 12-min, or an 18-min variable-interval tape, or food was removed completely (EXT). The order and frequency of free food delivery is indicated on the abscissa of Figure 1.

Table 1 gives the mismatching rates and mismatching accuracy for the three birds over the initial presentation of the 2-min, 6-min, 12-min, and 18-min variable-interval values of free grain delivery. Each schedule of free grain delivery was replicated several times. Figure 1 shows the results obtained over the 15 grain frequency conditions of the experiment for each of the three birds. Figure 1 presents the rate of mismatching sequences obtained under both the CR component and the non-CR component. The rate of incorrect matching sequences for both components over the 15 grain frequency conditions is shown for one bird, 27B. Each plotted point is the median rate based on the final five sessions under each of the grain frequency conditions. The dashed vertical line in Figure 1 represents a change from 90-min to 50-min sessions for each subject. This change was necessitated by procedural changes in the laboratory.

The results obtained in this experiment are similar to those of the previous experiment. Both Table 1 and Figure 1 show that in the case of each bird, mismatching rates were higher in the $\mathrm{CR}$ component than in the non-CR component under all the grain frequencies tested. Table 1 also shows that accuracy did not vary in any systematic way when free grain frequency was increased or decreased.

Figure 1 also shows an inverse relationship between the frequency of free food delivery and the rate of mismatching sequences. In general, the highest rates of 


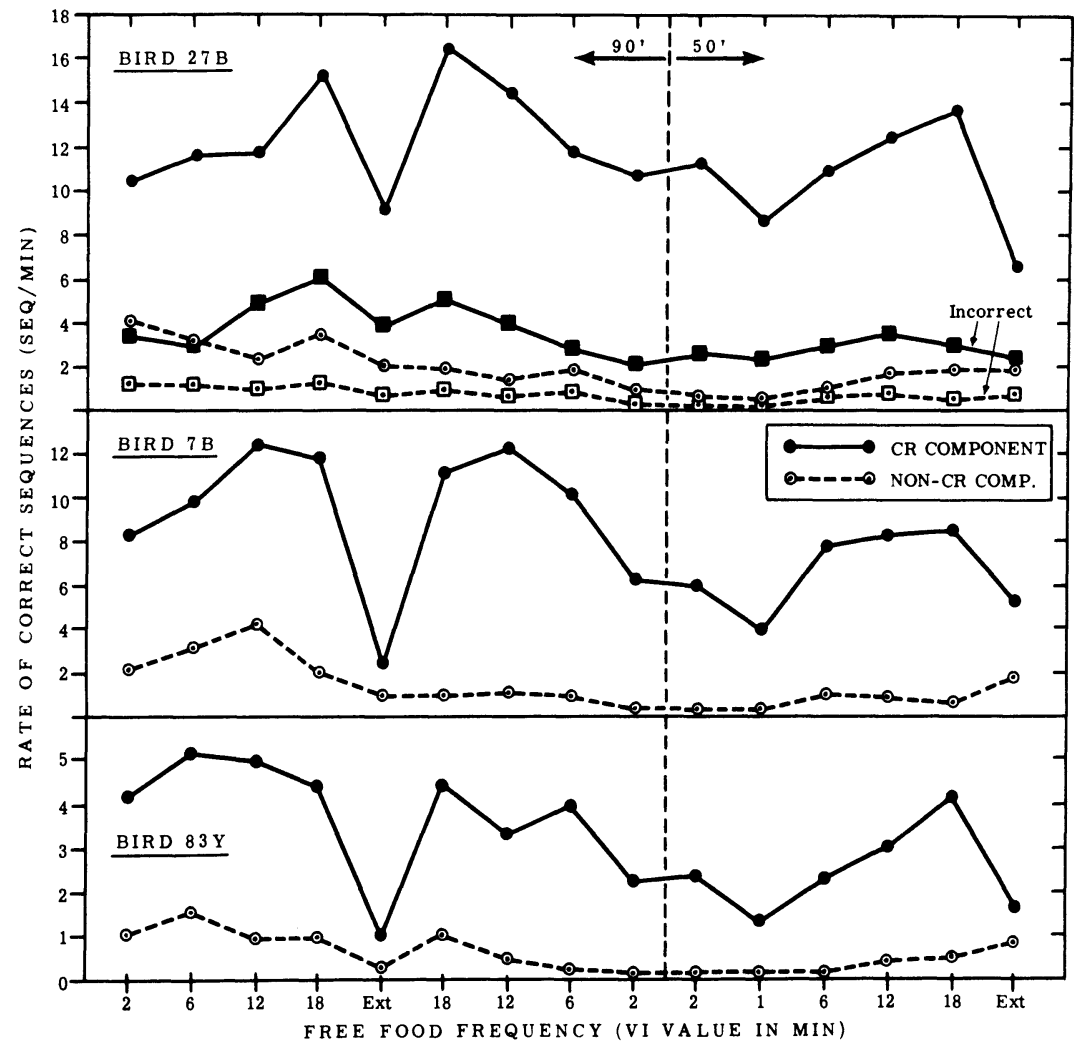

Figure 1. Rate of correct (mismatching) sequences in each component as a function of grain frequency. Incorrect (matching) sequence rates are also presented for Bird 27B. CR-conditioned reinforcement; EXT-food is absent. Vertical line indicates a change in session duration from 90 to $50 \mathrm{~min}$. mismatching were found under the 12-min and 18-min values of free food delivery, and the lowest rates were associated with the 1-min and 2-min values. The rates associated with the 6-min value fell between these high and low rates. The low rates associated with the 1-min and EXT conditions are similar to those obtained by Zimmerman (1969). Zimmerman, however, did not find any systematic relationship between the frequency of free food delivery and the rate of responding for CR over the values $2 \mathrm{~min}$ to $18 \mathrm{~min}$. Since the two studies differ with respect to a number of variables, it is difficult to account for the differences.

The results of Experiment II systematically replicate those found in Experiment $I$ and extend the results of Experiment I to a wide range of free food frequencies. The results of both these experiments, furthermore, systematically replicate with a complex task, the results previously obtained in this laboratory with a simple keypecking task. That is, the present results again show that conditioned reinforcement can maintain behavior in pigeons. The results of Experiment II further suggest that the capacity of a CR to maintain behavior is not weakened by decreasing the frequency of CR pairings with the delivery of food over the range of frequencies of free food covered in this experiment. These results suggest that the strength of a conditioned reinforcer, once it has been established, may become semiautonomous from the primary reinforcer with which it was originally paired.

\section{REFERENCES}

Ferster, C. B. Intermittent reinforcement of matching-to-sample in the pigeon. Journal of Experimental Analysis of Behavior, $1960,3,259-272$

Leckrone, W. R., Zimmerman, J., \& Hanford, P. V. A pigeon magazine shutter for preventing unprogrammed grain reinforcement. Psy chonomic Science, 1966, 6, 239-240.

Zimmerman, J. Meanwhile, back at the key: Maintenance of behavior by conditioned reinforcement and response independent primary reinforcement. In D. P. Hendry (Ed.), Conditioned reinforcement. Homewood, Ill: Dorsey Press, 1969.

Zimmerman, J., \& Ferster, C. B. Intermittent punishment of S responding in matching to sample. Journal of Experimental Analysis of Behavior, 1963, 6, 349-356.

Zimmerman, J., \& Hanford, P. Sustaining behavior with conditioned reinforcement as the only response-produced consequence. Psy chological Reports, 1966, 19, 391-401. 